\title{
Solving isomorphism problems about 2-designs from disjoint difference families
}

\author{
Christian Kaspers* and Alexander Pott*
}

July 6, 2018

\begin{abstract}
Recently, two new constructions of $(v, k, k-1)$ disjoint difference families in Galois rings were presented by Davis, Huczynska, and Mullen [8] and Momihara [12]. Both were motivated by a well-known construction of difference families from cyclotomy in finite fields by Wilson [17]. It is obvious that the difference families in the Galois ring and the difference families in the finite field are not equivalent. A related question, which is in general harder to answer, is whether the associated designs are isomorphic or not. In our case, this problem was raised by the authors of [8]. In this paper we show that the $2-(v, k, k-1)$ designs arising from the difference families in Galois rings $[8,12]$ and those arising from the difference families in finite fields [17] are nonisomorphic by comparing their block intersection numbers.
\end{abstract}

Keywords disjoint difference family, Galois ring, combinatorial design, isomorphism problem, intersection number, cyclotomic number

\section{Introduction}

Various types of difference families have long been studied in combinatorial literature $[1,3,7,9,17]$. They have applications in coding theory, and communications and information security [13], and they have connections to many other combinatorial objects, in particular to combinatorial designs. When, like recently by Davis, Huczynska, and Mullen [8] and by Momihara [12], a new construction of difference families is presented, it is a natural question to ask whether the new construction also leads to new difference families. In this paper, we compare two infinite families of difference families in Galois rings $[8,12]$ with a well-known infinite family of difference families in finite fields which was introduced by Wilson [17] in 1972. Since the difference families we compare are in different groups, they cannot be equivalent. Hence, we will examine whether the

*Faculty of Mathematics, Otto von Guericke University Magdeburg, 39106 Magdeburg, Germany (email: christian.kaspers@ovgu.de, alexander.pott@ovgu.de) 
associated combinatorial designs are isomorphic or not. This question is of particular interest because the authors of both [8] and [12] mention that they were inspired by the construction of Wilson [17], and the authors of [8] state explicitly that it is not known in general if the associated designs are nonisomorphic.

We start by defining the relevant objects we examine in this paper. First, we need the following notations: Let $G$ be an abelian group, $A, B \subseteq G$ and $g \in G$. We define multisets

$$
\begin{aligned}
\Delta A & :=\left\{a-a^{\prime}: a, a^{\prime} \in A, a \neq a^{\prime}\right\}, \\
\Delta_{+} A & :=\left\{a+a^{\prime}: a, a^{\prime} \in A, a \neq-a^{\prime}\right\}, \\
A-B & :=\{a-b: a \in A, b \in B, a \neq b\}, \\
A+B & :=\{a+b: a \in A, b \in B, a \neq-b\}, \\
A+g & :=\{a+g: a \in A\} .
\end{aligned}
$$

In the course of this paper we will sometimes use these notations to denote sets, not multisets. It will be clear from the context if the multiset or the respective set is meant.

Definition 1. Let $G$ be an abelian group of order $v$, and let $D_{1}, D_{2}, \ldots, D_{b}$ be $k$-subsets of $G$. The collection $D=\left\{D_{1}, D_{2}, \ldots, D_{b}\right\}$ of these subsets is called a difference family in $G$ with parameters $(v, k, \lambda)$ if each nonzero element of $G$ occurs exactly $\lambda$ times in the multiset union

$$
\bigcup_{i=1}^{b} \Delta D_{i} .
$$

If the subsets $D_{1}, D_{2}, \ldots, D_{b}$ are mutually disjoint, they form a disjoint difference family. If $b=1$, one speaks of a $(v, k, \lambda)$ difference set. We call $D$ complete (or near-complete) if the $D_{i}$ partition $G$ (or $G \backslash\{0\}$, respectively).

In this paper we will focus on near-complete $(v, k, k-1)$ disjoint difference families. These objects are closely related to so-called external difference families:

Definition 2. Let $G$ be an abelian group of order $v$, and let $D_{1}, D_{2}, \ldots, D_{b}$ be mutually disjoint $k$-subsets of $G$. The collection $D=\left\{D_{1}, D_{2}, \ldots, D_{b}\right\}$ of these subsets is called a $(v, k, \lambda)$ external difference family if each nonzero element of $G$ occurs exactly $\lambda$ times in the multiset union

$$
\bigcup_{\substack{1 \leq i, j \leq b \\ i \neq j}}\left(D_{i}-D_{j}\right) .
$$

Analogously to Definition 1 we call an external difference family complete (or nearcomplete) if the $D_{i}$ partition the (nonzero) elements of $G$.

The following Proposition 1.1 shows that under certain conditions a disjoint difference family is also an external difference family. This result was observed by Momihara [12], and, for near-complete disjoint difference families, it was also mentioned by Chang and Ding [7] and Davis, Huczynska, and Mullen [8]. We will add a short proof. 
Proposition 1.1. Let $G$ be an abelian group of order $v$, and let $D=\left\{D_{1}, D_{2}, \ldots, D_{b}\right\}$ be $a(v, k, \lambda)$ disjoint difference family in $G$. The collection $D$ forms an external difference family in $G$ if and only if the union $\bigcup_{i=1}^{b} D_{i}$ of the $D_{i}$ is a $\left(v, b k, \lambda^{\prime}\right)$ difference set in $G$ for some integer $\lambda^{\prime}>\lambda$. As an external difference family, $D$ has parameters $\left(v, k, \lambda^{\prime}-\lambda\right)$.

Proof. Let $G$ be an abelian group of order $v$, and let $D=\left\{D_{1}, D_{2}, \ldots, D_{b}\right\}$ be a collection of mutually disjoint $k$-subsets whose union $\bigcup_{i=1}^{b} D_{i}$ is a $\left(v, b k, \lambda^{\prime}\right)$ difference set for some integer $\lambda^{\prime}>2$. We can split all the differences in $\bigcup_{i=1}^{b} D_{i}$ in the following way into the "internal" and the "external" differences of the $D_{i}$ :

$$
\Delta\left(\bigcup_{i=1}^{b} D_{i}\right)=\bigcup_{i=1}^{b} \Delta D_{i} \cup \bigcup_{\substack{1 \leq i, j \leq b \\ i \neq j}}\left(D_{i}-D_{j}\right) .
$$

Since $\bigcup_{i=1}^{b} D_{i}$ is a difference set, each element $g \in G \backslash\{0\}$ is represented as $\lambda^{\prime}$ differences in $\Delta\left(\bigcup_{i=1}^{b} D_{i}\right)$. It follows that each nonzero element in $G$ is represented as $\lambda$ differences in $\bigcup_{i=1}^{b} \Delta D_{i}$ (meaning $D$ is a $(v, k, \lambda)$ disjoint difference family) if and only if it is represented $\lambda^{\prime}-\lambda$ times in $\bigcup_{1 \leq i, j \leq b, i \neq j}\left(D_{i}-D_{j}\right)$ (meaning $D$ is a $\left(v, k, \lambda^{\prime}-\lambda\right)$ external difference family).

From Proposition 1.1 it follows that every near-complete $(v, k, k-1)$ disjoint difference family is also a near-complete $(v, k, v-k-1)$ external difference family because the $D_{i}$ partition $G \backslash\{0\}$ and $G \backslash\{0\}$ is a $(v, v-1, v-2)$ difference set in $G$. For extended background on $(v, k, k-1)$ disjoint difference families the reader is referred to Buratti [6] who gives an overview over these difference families and summarizes several constructions, including the one by Davis, Huczynska, and Mullen [8] (see section 5 for some additional information). As mentioned above, every difference family gives rise to a combinatorial design.

Definition 3. Let $P$ be a set with $v$ elements (points). A $t$ - $(v, k, \lambda)$ design (or $t$-design, in brief) is a collection of $k$-subsets (blocks) of $P$ such that each $t$-subset of $P$ is contained in exactly $\lambda$ blocks.

The designs coming from difference families are 2-designs, which are often referred to as balanced incomplete block designs (BIBD). They can be constructed from difference families in the following way.

Definition 4. Let $G$ be an abelian group, and let $D=\left\{D_{1}, D_{2}, \ldots, D_{b}\right\}$ be a family of subsets of $G$. The development $\operatorname{dev}(D)$ of $D$ is the collection

$$
\left\{D_{i}+g: D_{i} \in D, g \in G\right\}
$$

of all the translates of the subsets contained in $D$. The sets $D_{1}, D_{2}, \ldots, D_{b}$ are called the base blocks of $\operatorname{dev}(D)$.

In other words: The development $\operatorname{dev}(D)$ of $D$ contains the orbits of the sets $D_{i} \in D$ under the action of $G$. If all the orbits have full length, $\operatorname{dev}(D)$ consists of $v b$ blocks. The following Proposition 1.2 is well known. We will add a proof for completeness. 
Proposition 1.2. Led $D$ be $a(v, k, \lambda)$ difference family in an abelian group $G$. The development dev $(D)$ of $D$ forms a $2-(v, k, \lambda)$ design with point set $G$.

Proof. Let $D=\left\{D_{1}, D_{2}, \ldots, D_{b}\right\}$ be a $(v, k, \lambda)$ difference family in an abelian group $G$. Take an arbitrary 2-subset $\left\{t_{1}, t_{2}\right\}$ of $G$. We need to show that $\left\{t_{1}, t_{2}\right\}$ is contained in $\lambda$ blocks of $\operatorname{dev}(D)$. Let $d=t_{1}-t_{2}$. Since $d \neq 0, d$ is represented $\lambda$ times as a difference $d=d^{\prime}-d^{\prime \prime}$, where $d^{\prime}, d^{\prime \prime} \in D_{i}, 1 \leq i \leq b$. Obviously, the differences in a base block $D_{i}$ and in all its translates $D_{i}+g, g \in G$, are the same, in short: $\Delta D_{i}=\Delta\left(D_{i}+g\right)$ for all $1 \leq i \leq b$ and $g \in G$. Hence, for each of the $\lambda$ pairs $d^{\prime}, d^{\prime \prime}$ we choose $g$ such that $d^{\prime}+g=t_{1}$. Then $d^{\prime \prime}+g=t_{2}$ and consequently $\left\{t_{1}, t_{2}\right\} \subseteq D_{i}+g$.

\section{Disjoint difference families from cyclotomy in finite fields}

First, we present the well-known construction of disjoint difference families in finite fields by Wilson [17]. It makes use of the cyclotomoy of the $e$-th powers in a finite field. Let $q$ be a power of a prime $p$. We denote by $\mathbb{F}_{q}$ the finite field with $q$ elements and by $\alpha$ a generator of the multiplicative group $\mathbb{F}_{q}^{*}$ of $\mathbb{F}_{q}$.

Theorem 2.1. Let $e, f$ be integers satisfying ef $=q-1, e, f \geq 2$, and let

$$
C_{i}=\left\{\alpha^{t} \mid t \equiv i \quad(\bmod e)\right\}, i=0,1, \ldots, e-1,
$$

be the cosets of the unique subgroup $C_{0}$ of index $e$ and order $f$ formed by the e-th powers of $\alpha$ in $\mathbb{F}_{q}^{*}$. Then, the family $C=\left\{C_{0}, C_{1}, \ldots, C_{e-1}\right\}$ of all these cosets forms a $(q, f, f-1)$ near-complete disjoint difference family in the additive group $\left(\mathbb{F}_{q},+\right)$.

Proof. This proof is similar to the proof by Davis, Huczynska, and Mullen [8, Theorem 2.1] where the authors prove that $C$ is a near-complete external difference family (see Proposition 1.1). Let $x, y \in \mathbb{F}_{q}^{*}$, and let $z=y x^{-1}$. Suppose $x=c-c^{\prime}$ for $c, c^{\prime} \in C_{i}, 0 \leq i \leq e-1$. Then $y=z x=z c-z c^{\prime}$ and, obviously, $z c, z c^{\prime}$ are in the same coset $C_{j}$. Hence, we have found a representation of $y$ as the difference of two distinct elements from the same set $C_{j}$. The other way around, every difference for $y$ will give us a difference for $x$. Consequently, every element of $\mathbb{F}_{q}^{*}$ will have the same number of differences. So, all we need to do is to count the total number of differences and divide it by the number of elements in $\mathbb{F}_{q}^{*}$ : There are $e$ sets $C_{i}$, and in each $C_{i}$ we can calculate $f(f-1)$ differences, giving us a total of $e f(f-1)$ differences. In $\mathbb{F}_{q}^{*}$ there are $q-1=e f$ elements. Hence, each element $x \in \mathbb{F}_{q}^{*}$ will have

$$
\frac{e f(f-1)}{e f}=f-1
$$

differences $x=c-c^{\prime}$ where $c$ and $c^{\prime}$ come from the same set $C_{i}$.

We remark that this theorem can also be proved using the results of Furino [9, Theorem 3.3, Corollary 3.5]. Employing the construction of a 2-design mentioned above, the development $\operatorname{dev}(C)$ of $C$ is a $2-(q, f, f-1)$ design. 


\section{Galois rings}

In this section, we will give a short introduction to Galois rings, see the work by Wan [16] for extended general background on this topic. Let $p$ be a prime, and let $f(x) \in \mathbb{Z}_{p^{m}}[x]$ be a monic basic irreducible polynomial of degree $r$. The factor ring $\mathbb{Z}_{p^{m}}[x] /\langle f(x)\rangle$ is called a Galois ring of characteristic $p^{m}$ and extension degree $r$. It is denoted by $\operatorname{GR}\left(p^{m}, r\right)$, and its order is $p^{m r}$. Since any two Galois rings of the same characteristic and the same order are isomorphic, we will speak of the Galois ring $\operatorname{GR}\left(p^{m}, r\right)$.

Galois rings are local commutative rings. The unique maximal ideal of the ring $R:=$ $\operatorname{GR}\left(p^{m}, r\right)$ is $\mathcal{I}=p R:=\{p a: a \in R\}$. The factor ring $R / \mathcal{I}$ is isomorphic to the finite field $\mathbb{F}_{p^{r}}$ with $p^{r}$ elements. As a system of representatives of $R / \mathcal{I}$ we take the Teichmüller set $\mathcal{T}=\left\{0,1, \xi, \ldots, \xi^{p^{r}-2}\right\}$ where $\xi$ denotes a root of order $p^{r}-1$ of $f(x)$. It is convenient to choose the generalized Conway polynomial, i. e. the Hensel lift from $\mathbb{F}_{p}[x]$ to $\mathbb{Z}_{p^{m}}[x]$ of the Conway polynomial, as our polynomial $f(x)$ since then, $x+(f)$ is a generator of the Teichmüller group, and we set $\xi=x+(f)$ (see [18, Section 1.3] for more information on the generalized Conway polynomial and its construction). An arbitrary element $a$ of $R$ has a unique $p$-adic representation $a=\alpha_{0}+p \alpha_{1}+\cdots+p^{m-1} \alpha_{m-1}$, where $\alpha_{0}, \alpha_{1}, \ldots, \alpha_{m-1} \in \mathcal{T}$.

The elements of $R \backslash \mathcal{I}$ are all the units of $R$, this unit group is denoted by $R^{*}$. It has order $p^{m r}-p^{(m-1) r}=p^{(m-1) r}\left(p^{r}-1\right)$ and is the direct product of the cyclic Teichmüller group $\mathcal{T}^{*}=\mathcal{T} \backslash\{0\}=\left\{1, \xi, \ldots, \xi^{p^{r}-2}\right\}$ of order $p^{r}-1$ and the group of principal units $\mathbb{P}:=1+\mathcal{I}$ of order $p^{(m-1) r}$. If $p$ is odd or if $p=2$ and $m \leq 2$, then $\mathbb{P}$ is a direct product of $r$ cyclic groups of order $p^{m-1}$. If $p=2$ and $m \geq 3$, then $\mathbb{P}$ is a direct product of a cyclic group of order 2, a cyclic group of order $2^{m-2}$ and $r-1$ cyclic groups of order $2^{m-1}$. So we have $R^{*}=\mathcal{T}^{*} \times \mathbb{P}$. In this paper, we will only consider Galois rings of characteristic $p^{2}$. In this case, $(1+p \alpha)(1+p \beta)=1+p(\alpha+\beta)$ for any $\alpha, \beta \in \mathcal{T}$, and every unit $u \in \operatorname{GR}\left(p^{2}, r\right)^{*}$ has a unique representation $u=\alpha_{0}\left(1+p \alpha_{1}\right), \alpha_{0}, \alpha_{1} \in \mathcal{T}, \alpha_{0} \neq 0$. Moreover, if $m=2$, the group of principal units $\mathbb{P}$ is a direct product of $r$ cyclic groups of order $p$ and thus has the structure of an elementary abelian group of order $p^{r}$.

\section{Disjoint difference families in Galois rings I}

We will now present the new construction of disjoint difference families in Galois rings $\operatorname{GR}\left(p^{2}, 2 n\right)$ with characteristic $p^{2}$ and even degree $r=2 n, n \in \mathbb{N}$, that was introduced by Momihara [12]: Let $p$ be a prime. Let $R_{2 n}$ denote the Galois ring $\operatorname{GR}\left(p^{2}, 2 n\right)=$ $\mathbb{Z}_{p^{2}}[x] /\langle f(x)\rangle$, where $f(x)$ is a monic basic irreducible polynomial of degree $2 n$, and let $\xi$ be a root of order $p^{2 n}-1$ of $f(x)$. Let $\mathcal{I}_{2 n}$ be the maximal ideal and let $\mathbb{P}_{2 n}=1+\mathcal{I}_{2 n}$ be the group of principal units of $R_{2 n}$. Moreover, we have the Teichmüller set

$$
\mathcal{T}_{2 n}=\left\{0,1, \xi, \ldots, \xi^{p^{2 n}-2}\right\}
$$

and each element of $R_{2 n}$ has a unique $p$-adic representation

$$
a_{0}+p a_{1}, \quad a_{0}, a_{1} \in \mathcal{T}_{2 n} .
$$


The Galois ring $R_{2 n}$ contains a unique Galois ring $R_{n}=\mathrm{GR}\left(p^{2}, n\right)$ of characteristic $p^{2}$ and degree $n$ as its subring [16, Theorem 14.24]. It can be constructed in the following way [16, Corollary 14.28]: Obviously, $\xi^{\left(p^{n}+1\right)}$ is a root of order $p^{n}-1$ of $f(x)$. It follows that

$$
\mathcal{T}_{n}=\left\{0,1, \xi^{p^{n}+1}, \xi^{2\left(p^{n}+1\right)}, \ldots, \xi^{\left(p^{n}-2\right)\left(p^{n}+1\right)}\right\}
$$

is the Teichmüller set of $R_{n}$ and $R_{n}=\left\{a_{0}+p a_{1}: a_{0}, a_{1} \in \mathcal{T}_{n}\right\}$. Then,

$$
R_{n}^{*}=\left\{\alpha_{0}\left(1+p \alpha_{1}\right): \alpha_{0}, \alpha_{1} \in \mathcal{T}_{n}, \alpha_{0} \neq 0\right\}
$$

is the unit group of the subring $R_{n}$. Analogously to $R_{2 n}$, let $\mathcal{I}_{n}$ denote the maximal ideal and $\mathbb{P}_{n}$ the group of principal units of $R_{n}$. So we have $R_{n}^{*}=\mathcal{T}_{n}^{*} \times \mathbb{P}_{n}$. Now, let $p S$ be a system of representatives of $\mathcal{I}_{2 n} / \mathcal{I}_{n}$ which means that $1+p S$ will be a system of representatives of $\mathbb{P}_{2 n} / \mathbb{P}_{n}$. Each element of $1+p S$ can be written as $1+p x$ for some $x \in \mathcal{T}_{2 n}$, i. e. $1+p S=\{1+p x: x \in S\}$ for some subset $S$ of $\mathcal{T}_{2 n}$. Write $S=\left\{x_{0}, x_{1}, \ldots, x_{p^{n}-1}\right\}$. Finally, define a coset $P$ of the maximal ideal $\mathcal{I}_{n}$ of $R_{n}$ as

$$
P=\left\{p \xi^{p^{n}}, p \xi^{\left(p^{n}+1\right)+p^{n}}, p \xi^{2\left(p^{n}+1\right)+p^{n}}, \ldots, p \xi^{\left(p^{n}-2\right)\left(p^{n}+1\right)+p^{n}}\right\} .
$$

Theorem 4.1 ([12, Theorem 1]). Using the notation from above, define subsets

$$
D_{i}=\xi^{i}\left(P \cup\left(\bigcup_{j=0}^{p^{n}-1} \xi^{j}\left(1+p x_{j}\right) R_{n}^{*}\right)\right), i=0,1, \ldots, p^{n},
$$

of $R_{2 n}$. The family $D=\left\{D_{0}, D_{1}, \ldots, D_{p^{n}}\right\}$ forms a near-complete disjoint difference family in $\left(R_{2 n},+\right)$ with parameters $\left(p^{4 n},\left(p^{2 n}+1\right)\left(p^{n}-1\right),\left(p^{2 n}+1\right)\left(p^{n}-1\right)-1\right)$.

For the extensive and technical proof of this theorem the reader is referred to Momihara [12]. The author of [12] mentions that, since $\left(p^{2 n}+1\right)\left(p^{n}-1\right)$ divides $p^{4 n}-1$, we can use Theorem 2.1 to construct a disjoint difference family $C=\left\{C_{0}, C_{1}, \ldots, C_{p^{n}}\right\}$ with the same parameters as in Theorem 4.1 in the additive group of the finite field $\mathbb{F}_{p^{4 n}}$. According to Proposition 1.2 the developments $\operatorname{dev}(C)$ and $\operatorname{dev}(D)$ of the difference families form 2-designs with parameters $(v, k, \lambda)=\left(p^{4 n},\left(p^{2 n}+1\right)\left(p^{n}-1\right),\left(p^{2 n}+1\right)\left(p^{n}-1\right)-1\right)$. The comparison of these two 2-designs leads to our first main theorem:

Theorem 4.2. Let $C$ be a $\left(p^{4 n},\left(p^{2 n}+1\right)\left(p^{n}-1\right),\left(p^{2 n}+1\right)\left(p^{n}-1\right)-1\right)$ disjoint difference family in the additive group of the finite field $\mathbb{F}_{p^{4 n}}$ constructed with Theorem 2.1, and let $D$ be a disjoint difference families with the same parameters in the additive group of the Galois ring $G R\left(p^{2}, 2 n\right)$ constructed with Theorem 4.1. The 2- $(v, k, k-1)$ designs $\operatorname{dev}(C)$ and $\operatorname{dev}(D)$ with parameters $v=p^{4 n}$ and $k=\left(p^{2 n}+1\right)\left(p^{n}-1\right)$ are nonisomorphic.

There are various ways of isomorphism testing for combinatorial designs. One popular approach is to study the ranks of their incidence matrices. However, in our case this yields no valid results. We were more successful examining the so-called block intersection numbers of both 2-designs. We will prove Theorem 4.2 by showing that the block intersection numbers of $\operatorname{dev}(C)$ and $\operatorname{dev}(D)$ differ. The block intersection numbers of a 
$t$-design are the cardinalities $\left|B_{i} \cap B_{j}\right|$ of the intersections of two distinct blocks $B_{i}, B_{j}$ of the design.

Remark 1. Block intersection numbers can be easily calculated in the following way: Let $M$ denote the incidence matrix of a $t$-design with the rows of $M$ corresponding to the points and the columns of $M$ corresponding to the blocks of the design. The entry $(i, j)$ of the matrix $M^{T} M$ is exactly $\left|B_{i} \cap B_{j}\right|$.

Block intersection numbers of combinatorial designs are invariant under isomorphism. So, to prove that our designs are nonisomorphic it is sufficient to show that $\operatorname{dev}(D)$ has one block intersection number different from the block intersection numbers of $\operatorname{dev}(C)$.

We will first calculate all the intersection numbers of $\operatorname{dev}(C)$. These are given as the so-called cyclotomic numbers: Analogously to section 2 , let $C_{0}, C_{1}, \ldots, C_{e-1}$ be the cosets of the subgroup $C_{0}$ of the $e$-th powers in $\mathbb{F}_{q}^{*}$. For fixed non-negative integers $i, j \leq e-1$ the cyclotomic number $(i, j)_{e}$ of order $e$ is defined as

$$
(i, j)_{e}=\left|\left(C_{i}+1\right) \cap C_{j}\right| .
$$

In general, it is a hard number theoretic problem to calculate these cyclotomic numbers. However, Baumert, Mills, and Ward [2] proved that in special cases they are easy to calculate:

Proposition 4.3 ([2, Theorems 1 and 4$])$. Let $p$ be a prime, and let $e \geq 3$ be a divisor of $p^{m}-1$ for a positive integer $m$. If -1 is a power of $p$ modulo $e$, then either $p=2$ or $f=\left(p^{m}-1\right) / e$ is even, $p^{m}=s^{2}$ and $s \equiv 1(\bmod e)$, and the cyclotomic numbers of order e are given as

$$
\begin{aligned}
(0,0)_{e} & =\eta^{2}-(e-3) \eta-1, & & \\
(0, i)_{e}=(i, 0)_{e}= & (i, i)_{e}=\eta^{2}+\eta & & \text { for } i \neq 0, \\
& (i, j)_{e}=\eta^{2} & & \text { for } i \neq j \text { and } i, j \neq 0,
\end{aligned}
$$

where $\eta=(s-1) / e$.

Because there exist only three distinct cyclotomic numbers in the described case, the authors of [2] speak of uniform cyclotomic numbers. Applying Proposition 4.3 to $\operatorname{dev}(D)$ leads to

Corollary 4.4. Let $C$ be a $\left(p^{4 n},\left(p^{2 n}+1\right)\left(p^{n}-1\right),\left(p^{2 n}+1\right)\left(p^{n}-1\right)-1\right)$ disjoint difference family in the additive group of the finite field $\mathbb{F}_{p^{4 n}}$ constructed with Theorem 2.1. The $2-\left(p^{4 n},\left(p^{2 n}+1\right)\left(p^{n}-1\right),\left(p^{2 n}+1\right)\left(p^{n}-1\right)-1\right)$ design dev $(C)$ has exactly three block intersection numbers, namely $p^{n}-2, p^{n}\left(p^{n}-1\right)$ and $\left(p^{n}-1\right)^{2}$.

Proof. We show that $C$ meets the conditions of Proposition 4.3: In our case $e=p^{n}+1$. So -1 is the $n$-th power of $p$ modulo $e$. Moreover, from $p^{4 n}=s^{2}$ it follows that $s=p^{2 n} \equiv 1$ 
$(\bmod e)$, so $\eta=\left(p^{2 n}-1\right) /\left(p^{n}+1\right)=p^{n}-1$. By (1) we obtain the cyclotomic numbers

$$
\begin{aligned}
(0,0)_{p^{n}+1} & =p^{n}-2, & & \\
(0, i)_{p^{n}+1}=(i, 0)_{p^{n}+1}=(i, i)_{p^{n}+1} & =p^{n}\left(p^{n}-1\right) & & \text { for } i \neq 0, \\
(i, j)_{p^{n}+1} & =\left(p^{n}-1\right)^{2} & & \text { for } i \neq j \text { and } i, j \neq 0,
\end{aligned}
$$

that occur as the intersection numbers of $\operatorname{dev}(C)$.

The next step will be to show that there is a block intersection number in $\operatorname{dev}(D)$ that does not occur in $\operatorname{dev}(C)$.

Lemma 4.5. Let $D$ be a $\left(p^{4 n},\left(p^{2 n}+1\right)\left(p^{n}-1\right),\left(p^{2 n}+1\right)\left(p^{n}-1\right)-1\right)$ disjoint difference family in the additive group of $G R\left(p^{2}, 2 n\right)$ constructed with Theorem 4.1. The $2-\left(p^{4 n},\left(p^{2 n}+1\right)\left(p^{n}-1\right),\left(p^{2 n}+1\right)\left(p^{n}-1\right)-1\right)$ design dev $(D)$ has a block intersection number $\left(2 p^{n}-1\right)\left(p^{n}-2\right)$.

Proof. This proof has a similar structure to the proofs by Momihara [12, Lemmata 4 7], but unlike Momihara [12], we will not consider all the sets $D_{0}, D_{1}, \ldots, D_{b}$ of the difference family, but only $D_{0}$. This requires a more detailed analysis of the intersection relations.

Let, like above, $\xi$ be a generator of the Teichmüller group, $x_{j} \in S$, where $1+p S$ is a system of representatives of $\mathbb{P}_{2 n} / \mathbb{P}_{n}$, and $R_{n}^{*}$ denote the unit group of the subring $R_{n}=\operatorname{GR}\left(p^{2}, n\right)$. Furthermore, define subsets $U$ and $V$ of $R_{2 n}^{*}$ as

$$
U=\bigcup_{j=0}^{p^{n}-1} \xi^{j}\left(1+p x_{j}\right) R_{n}^{*} \quad \text { and } \quad V=\bigcup_{j=0}^{p^{n}-1}\left(1+p x_{j}\right) R_{n}^{*} .
$$

Note that $D_{i}=\xi^{i}(P \cup U)$ and that $V=\mathcal{T}_{n}^{*} \times \mathbb{P}_{2 n}$ and $\bigcup_{j=0}^{p^{n}} \xi^{j} V=R_{2 n}^{*}$. We will prove Lemma 4.5 by showing that the block intersection number $\left|\left(D_{0}+u\right) \cap D_{0}\right|$ of the block $D_{0}$ and its translate $D_{0}+u$ equals $\left(2 p^{n}-1\right)\left(p^{n}-2\right)$ for all $u \in U$. The above statement is equivalent to: An arbitrary element $u \in U$ occurs exactly $\left(2 p^{n}-1\right)\left(p^{n}-2\right)$ times in the multiset $\Delta D_{0}$. Looking at the structure of

$$
D_{0}=P \cup U=P \cup\left(\bigcup_{j=0}^{p^{n}-1} \xi^{j}\left(1+p x_{j}\right) R_{n}^{*}\right),
$$

we can split all the differences in this multiset into four different types. Let $0 \leq s, t \leq$ $p^{n}-1$ and $s \neq t$. We have differences of

Type 1: $\quad \xi^{s}\left(1+p x_{s}\right) R_{n}^{*}-\xi^{t}\left(1+p x_{t}\right) R_{n}^{*}$,

Type 2: $\Delta\left(\xi^{s}\left(1+p x_{s}\right) R_{n}^{*}\right)$,

Type 3: $\quad \xi^{s}\left(1+p x_{s}\right) R_{n}^{*}-P$,

Type 4: $\triangle P$. 
Now, let $u$ be a fixed element of $U$. We will count the number of occurrences of $u$ in $\Delta D_{0}$ by counting its occurrences in each of the four multisets defined above. Before we start we state the following useful lemma which will show that it does not matter whether we look at the differences or the sums in type 1-type 4:

Lemma 4.6. Consider the Galois Ring $G R\left(p^{m}, r\right)$. If $p$ is odd, then -1 is an element of the Teichmüller group $\mathcal{T}^{*}$. If $p=2$, then -1 is a principal unit.

Proof. Let $p$ be odd. Then, the group of principal units $\mathbb{P}$ is a direct product of $r$ cyclic groups, each of odd order $p^{m-1}$, and the Teichmüller group $\mathcal{T}^{*}$ has even order $p^{r}-1$. Consequently, there are only two second roots of unity in $\operatorname{GR}\left(p^{m}, r\right): 1$ and -1 . Hence, $-1=\xi^{\left(p^{r}-1\right) / 2} \in \mathcal{T}^{*}$. If $p=2$, all the even integers of $\mathbb{Z}_{p^{m}} \subseteq \operatorname{GR}\left(p^{m}, r\right)$ are elements of the maximal ideal $\mathcal{I}=2 R$. Since -1 is odd, it follows that $-1 \in \mathbb{P}=1+\mathcal{I}$.

In our case, $m=2$ and $r=2 n$, it follows from Lemma 4.6 that if $p$ is odd, $-1=$ $\xi^{\left(p^{2 n}-1\right) / 2}=\xi^{\left(p^{n}-1\right)\left(p^{n}+1\right) / 2}$, and thus -1 is included in the Teichmüller group $\mathcal{T}_{n}^{*}=$ $\left\{\xi^{i\left(p^{n}+1\right)}: i=0,1, \ldots, p^{n}-2\right\}$ of the subring $R_{n}$. Because $\mathcal{T}_{n}^{*} \subseteq R_{n}^{*}$ and $P=p \xi^{p^{n}} \mathcal{T}_{n}^{*}$, we have

$$
R_{n}^{*}=-R_{n}^{*}, \quad \text { and } \quad P=-P .
$$

If $p=2$, we have $-1=3=1 \cdot(1+2 \cdot 1) \in R_{n}^{*}$, since clearly $1 \in \mathcal{T}_{n}^{*}$. Furthermore, since $-2=2$, all the elements of $\mathcal{I}_{2 n}=2 R_{2 n}$ (and consequently of $P \subseteq \mathcal{I}_{2 n}$ ) are self inverse. Thus, (2) holds in this case as well. Now we start our proof by analyzing differences of type 1 , and we first state a helpful lemma [12]:

Lemma 4.7 ([12, Lemma 3]). Let $a$ be an integer, $0 \leq a \leq p^{n}-2$, let $b$ be an element of $R_{2 n}$, and let $V$ be as defined above. If $\xi^{a}(1+p b) \notin R_{n}^{*}$ and $\xi^{a} \notin \mathcal{T}_{n}$, then

$$
R_{n}^{*}+\xi^{a}(1+p b) R_{n}^{*}=R_{2 n}^{*} \backslash\left(V \cup \xi^{a} V\right) .
$$

Now, let $0 \leq s, t \leq p^{n}-1, s \neq t$ be fixed. By (2) we have

$$
\xi^{s}\left(1+p x_{s}\right) R_{n}^{*}-\xi^{t}\left(1+p x_{t}\right) R_{n}^{*}=\xi^{s}\left(1+p x_{s}\right) R_{n}^{*}+\xi^{t}\left(1+p x_{t}\right) R_{n}^{*} .
$$

We factor out $\xi^{s}\left(1+p x_{s}\right)$ and obtain

$$
\xi^{s}\left(1+p x_{s}\right)\left(R_{n}^{*}+\xi^{t-s}\left(1+p\left(x_{t}-x_{s}\right)\right) R_{n}^{*}\right) .
$$

Applying Lemma 4.7, this equals

$$
\xi^{s}\left(1+p x_{s}\right)\left(R_{2 n}^{*} \backslash\left(V \cup \xi^{t-s} V\right)\right) .
$$

Since $(1+p x) V=V$ for any $x \in R_{2 n}$, the factor $\left(1+p x_{s}\right)$ can be omitted, and we obtain the result

$$
\xi^{s}\left(1+p x_{s}\right) R_{n}^{*}-\xi^{t}\left(1+p x_{t}\right) R_{n}^{*}=R_{2 n}^{*} \backslash\left(\xi^{s} V \cup \xi^{t} V\right)
$$


Now, we are able to count differences: The set $D_{0}$ contains $p^{n}$ distinct subsets of the type $\xi^{s}\left(1+p x_{s}\right) R_{n}^{*}$. Consequently, we have $p^{n}\left(p^{n}-1\right)$ type 1 multisets. Since $0 \leq s, t \leq p^{n}-1$, the elements of $\xi^{p^{n}} V$ are, according to (3), contained in each of these multisets, whereas the elements of $\bigcup_{j=0}^{p^{n}-1} \xi^{j} V=R_{2 n}^{*} \backslash \xi^{p^{n}} V$ occur in only $\left(p^{n}-2\right)\left(p^{n}-1\right)$ of them. Since $U$ is a subset of $R_{2 n}^{*} \backslash \xi^{p^{n}} V$, we count, so far, $\left(p^{n}-2\right)\left(p^{n}-1\right)$ occurrences of our element $u$.

In the next step, we will address differences of type 2 . We will argue similarly to Momihara [12, Lemma 6]. Let $s$ be a fixed integer, $0 \leq s \leq p^{n}-1$. We consider the set

$$
\Delta\left(\xi^{s}\left(1+p x_{s}\right) R_{n}^{*}\right)=\left\{\xi^{s}\left(1+p x_{s}\right) a-\xi^{s}\left(1+p x_{s}\right) b: a, b \in R_{n}^{*}, a \neq b\right\} .
$$

Since $a, b \in R_{n}^{*}$, we write $a=\xi^{a_{1}}\left(1+p a_{2}\right)$ and $b=\xi^{b_{1}}\left(1+p b_{2}\right)$, where $a_{1}, b_{1} \in\left\{j\left(p^{n}+1\right)\right.$ : $\left.j=0,1, \ldots, p^{n}-2\right\}, a_{2}, b_{2} \in \mathcal{T}_{n}$, and $\left(a_{1}, a_{2}\right) \neq\left(b_{1}, b_{2}\right)$. We will consider two cases. First, if $a_{1}=b_{1}$, we have

$$
\xi^{s}\left(1+p x_{s}\right) \xi^{a_{1}}\left(1+p a_{2}\right)-\xi^{s}\left(1+p x_{s}\right) \xi^{a_{1}}\left(1+p b_{2}\right) .
$$

We factor out $\xi^{s+a_{1}}$ and multiply the elements of $\mathbb{P}_{n}$ :

$$
\xi^{s+a_{1}}\left(1+p\left(x_{s}+a_{2}\right)-\left(1+p\left(x_{s}+b_{2}\right)\right)\right) .
$$

We summarize this expression and obtain

$$
\xi^{s+a_{1}} p\left(a_{2}-b_{2}\right)
$$

which is clearly an element of the maximal ideal $\mathcal{I}_{2 n}$. Thus, the case $a_{1}=b_{1}$ leads to no additional differences representing the unit $u$. Now, let $a_{1} \neq b_{1}$. Instead of (4), we now have

$$
\xi^{s}\left(1+p x_{s}\right) \xi^{a_{1}}\left(1+p a_{2}\right)-\xi^{s}\left(1+p x_{s}\right) \xi^{b_{1}}\left(1+p b_{2}\right) .
$$

Factoring out $\xi^{s}\left(1+p x_{s}\right)$ yields

$$
\xi^{s}\left(1+p x_{s}\right)\left(\xi^{a_{1}}\left(1+p a_{2}\right)-\xi^{b_{1}}\left(1+p b_{2}\right)\right),
$$

which equals

$$
\xi^{s}\left(1+p x_{s}\right)\left(\xi^{a_{1}}-\xi^{b_{1}}+p\left(\xi^{a_{1}} a_{2}-\xi^{b_{1}} b_{2}\right)\right) .
$$

Because $\xi^{a_{1}}-\xi^{b_{1}} \neq 0$, and $\xi^{a_{1}}, \xi^{b_{1}}, a_{2}, b_{2} \in R_{n}$, the terms $\xi^{a_{1}}-\xi^{b_{1}}+p\left(\xi^{a_{1}} a_{2}-\xi^{b_{1}} b_{2}\right)$ represent the elements of $R_{n}^{*}=R_{n} \backslash \mathcal{I}_{n}$. The unit group $R_{n}^{*}$ contains $p^{n}\left(p^{n}-1\right)$ elements, and we can choose $a_{1}, a_{2}, b_{1}, b_{2}$ in $p^{2 n}\left(p^{n}-1\right)\left(p^{n}-2\right)$ different ways. The differences are evenly distributed, which means that our element $u$ has $p^{n}\left(p^{n}-2\right)$ distinct representations of type 2 .

By adding our numbers of type 1 and type 2 difference representations of $u$ we have already reached the number $\left(2 p^{n}-1\right)\left(p^{n}-2\right)$ stated in Lemma 4.5. So, for the remaining 
types, we need to show that $u$ does not occur in multisets of of type 3 and type 4 . We examine differences of type $3: \xi^{s}\left(1+p x_{s}\right) R_{n}^{*}-P$. First, we take arbitrary elements $\xi^{k\left(p^{n}+1\right)}(1+p a), a \in \mathcal{T}_{n}$, from $R_{n}^{*}$ and $-p \xi^{\ell\left(p^{n}+1\right)+p^{n}}$ from $P$ (recall that $P=-P$ ), where $k, \ell \in\left\{0,1, \ldots, p^{n}-2\right\}$ and $a \in \mathcal{T}_{n}$. So, we are interested in differences of the form

$$
\xi^{s}\left(1+p x_{s}\right) \xi^{k\left(p^{n}+1\right)}(1+p a)+p \xi^{\ell\left(p^{n}+1\right)+p^{n}}
$$

where $s \in\left\{0,1, \ldots, p^{n}-1\right\}$ and $x_{s} \in S$ are fixed. We factor out $\xi^{s+k\left(p^{n}+1\right)}$, summarize, and obtain

$$
\xi^{s+k\left(p^{n}+1\right)}\left(1+p x_{s}\right)(1+p a)\left(1+p \xi^{(\ell-k)\left(p^{n}+1\right)+p^{n}-s}\right) .
$$

We write (5) with respect to all $0 \leq k, \ell \leq p^{n}-2$ and all $a \in \mathcal{T}_{n}$ :

$$
\xi^{s}\left(1+p x_{s}\right)\left(\mathcal{T}_{n}^{*} \mathbb{P}_{n}\left(1+p \xi^{p^{n}-s} \mathcal{T}_{n}^{*}\right)\right)
$$

Since $\xi^{p^{n}-s} \notin \mathcal{T}_{n}$, it is clear that $\mathbb{P}_{n}$ and $1+p \xi^{p^{n}-s} \mathcal{T}_{n}^{*}$ are disjoint. We show that each of the $p^{n}-1$ other cosets in $\mathbb{P}_{2 n} / \mathbb{P}_{n}$ is represented exactly once by $1+p \xi^{p^{n}-s} \mathcal{T}_{n}^{*}$. This is equivalent to showing that $p \xi^{p^{n}-s} \mathcal{T}_{n}$ represents every coset of $\mathcal{I}_{2 n} / \mathcal{I}_{n}$ except $\mathcal{I}_{n}=p \mathcal{T}_{n}^{*}$ itself. Assume there were two elements from the same coset in $p \xi^{p^{n}-s} \mathcal{T}_{n}^{*}$, then their difference would be in $\mathcal{I}_{n}$. However, for two distinct integers $k, \ell$, the difference

$$
p \xi^{p^{n}-s+k\left(p^{n}+1\right)}-\left(p \xi^{p^{n}-s+\ell\left(p^{n}+1\right)}\right)=\xi^{p^{n}-s}\left(p \xi^{k\left(p^{n}+1\right)}-p \xi^{\ell\left(p^{n}+1\right)}\right)
$$

is not in $\mathcal{I}_{n}$ since $\xi^{p^{n}-s} \notin \mathcal{T}_{n}$ and $\left(p \xi^{k\left(p^{n}+1\right)}-p \xi^{\ell\left(p^{n}+1\right)}\right) \in \mathcal{I}_{n}$. It follows that (6) equals

$$
\xi^{s}\left(1+p x_{s}\right)\left(\mathcal{T}_{n}^{*}\left(\mathbb{P}_{2 n} \backslash \mathbb{P}_{n}\right)\right) .
$$

With the definition of $V=\mathcal{T}_{n}^{*} \times \mathbb{P}_{2 n}$ from above, we have

$$
\xi^{s}\left(1+p x_{s}\right) R_{n}^{*}-P=\xi^{s}\left(V \backslash\left(1+p x_{s}\right) R_{n}^{*}\right) .
$$

Recall that $U=\bigcup_{j=0}^{p^{n}-1} \xi^{j}\left(1+p x_{j}\right) R_{n}^{*}$. Hence $u \in U$ does not occur in multisets of type 3 . We finish our proof by examining differences of type 4 : Since $P$ is a subset of the maximal ideal $\mathcal{I}_{2 n}$ the set of all the differences of distinct elements of $P$ is also a subset of $\mathcal{I}_{2 n}$. Thus, $\Delta P$ yields no representations of $u$.

For $p^{n}>2$, the intersection number $\left(2 p^{n}-1\right)\left(p^{n}-2\right)$ of $\operatorname{dev}(D)$ does not equal any of the cyclotomic numbers $p^{n}-2, p^{n}\left(p^{n}-1\right)$ and $\left(p^{n}-1\right)^{2}$ of $\operatorname{dev}(C)$. Hence, Corollary 4.4 in combination with Lemma 4.5 proves Theorem 4.2 for $p^{n}>2$. In the case $p^{n}=2$, i. e., $p=2$ and $n=1$, however, the intersection numbers of $\operatorname{dev}(C)$ match those from $\operatorname{dev}(D)$. We complete the proof of Theorem 4.2 by using the computer algebra system Magma [5] to compute the full automorphism groups of $\operatorname{dev}(C)$ and $\operatorname{dev}(D)$. We see that, for $p^{n}=2$, the automorphism group of $\operatorname{dev}(C)$ has order 960 , whereas for $\operatorname{dev}(D)$ it is 
only of order 192. Thus, these 2-(16,5,4) designs are nonisomorphic as well.

Remark 2. The full automorphism group of $\operatorname{dev}(D)$ has order $4 n p^{4 n}\left(p^{4 n}-1\right)$, it consists of the additive group $\left(\mathbb{F}_{p^{4 n}},+\right)$ of order $p^{4 n}$, the multiplicative group $\mathbb{F}_{p^{4 n}}^{*}$ of order $p^{4 n}-$ 1 and the Galois group $\operatorname{Gal}\left(\mathbb{F}_{p^{4 n}} / \mathbb{F}_{p}\right)$ of order $4 n$. The full automorphism group of $\operatorname{dev}(C)$ has order $2 p^{5 n\left(p^{2 n}-1\right)}$, it consists of the additive group $\left(R_{2 n},+\right)$ of order $p^{4 n}$, the Teichmüller group $\mathcal{T}_{2 n}$ of $R_{2 n}$ of order $p^{2 n}-1$, the group of principal units $\mathbb{P}_{n}$ of the subring $R_{n}$ of order $p^{n}$ and an interesting automorphism of order 2 of the additive group $\left(R_{2 n},+\right)$. In the case $p^{n}=2$, it is defined by $1 \mapsto 1+2 \xi, \xi \mapsto 1+3 \xi$.

\section{Disjoint difference families in Galois rings II}

Davis, Huczynska, and Mullen [8] found a new cyclotomic construction of near-complete $(v, k, k-1)$ external difference families in Galois rings $\operatorname{GR}\left(p^{2}, r\right)$ of characteristic $p^{2}$. This construction was in a more general way already given by Furino [9] in 1991 for arbitrary commutative rings with an identity. Furino [9] used the approach to create near-complete disjoint difference families, and we know from Proposition 1.1 that every near-complete disjoint difference family is also a near-complete external difference family. Furthermore, we remark that the construction by Furino [9] was generalized in the case $(v, k, k-1)$ by Buratti [6] to so-called Ferrero pairs $(G, A)$, where $A$ is a non-trivial group of automorphisms of $G$ acting semiregularly on a group $G \backslash\{0\}$. Before we state the result by Davis, Huczynska, and Mullen [8] we need the following two useful lemmas about differences in Galois rings. Let, like before, $\mathcal{T}$ denote the Teichmüller set, $\mathcal{T}^{*}=\mathcal{T} \backslash\{0\}$ denote the cyclic Teichmüller group having order $p^{r}-1$, and $\mathcal{I}=p \operatorname{GR}\left(p^{2}, r\right)$ denote the maximal ideal of the Galois ring $\operatorname{GR}\left(p^{2}, r\right)$.

Lemma 5.1. In $G R\left(p^{2}, r\right)$ the difference $u-u^{\prime}$ of two distinct units $u=\alpha_{0}\left(1+p \alpha_{1}\right), u^{\prime}=$ $\alpha_{0}^{\prime}\left(1+p \alpha_{1}^{\prime}\right)$, where $\alpha_{0}, \alpha_{0}^{\prime} \in \mathcal{T}^{*}, \alpha_{1}, \alpha_{1}^{\prime} \in \mathcal{T}$, is a unit if and only if $\alpha_{0} \neq \alpha_{0}^{\prime}$.

Proof. Let $u=\alpha_{0}\left(1+p \alpha_{1}\right), u^{\prime}=\alpha_{0}^{\prime}\left(1+p \alpha_{1}^{\prime}\right)$, where $\alpha_{0}, \alpha_{0}^{\prime} \in \mathcal{T}^{*}, \alpha_{1}, \alpha_{1}^{\prime} \in \mathcal{T}$. If $\alpha_{0}=\alpha_{0}^{\prime}=\alpha$, we have

$$
u-u^{\prime}=\alpha\left(1+p \alpha_{1}\right)-\alpha\left(1+p \alpha_{1}^{\prime}\right)=p \alpha\left(\alpha_{1}-\alpha_{1}^{\prime}\right),
$$

which is an element of $\mathcal{I}$. If $\alpha_{0} \neq \alpha_{0}^{\prime}$, we have

$$
u-u^{\prime}=\alpha_{0}\left(1+p \alpha_{1}\right)-\alpha_{0}^{\prime}\left(1+p \alpha_{1}^{\prime}\right)=\alpha_{0}-\alpha_{0}^{\prime}+p\left(\alpha_{1}-\alpha_{1}^{\prime}\right),
$$

which is clearly a unit since $\mathcal{T}$ is a system of representatives of $\operatorname{GR}\left(p^{2}, r\right) / \mathcal{I}$.

Lemma 5.2. 1. The multiset $\Delta \mathcal{T}^{*}$ contains only units of $G R\left(p^{2}, r\right)$.

2. Let $d \in(1+p \beta) \mathcal{T}^{*}$ for some $\beta \in \mathcal{T}^{*}$. If $d$ is contained in $\Delta \mathcal{T}^{*}$ then the whole coset $(1+p \beta) \mathcal{T}^{*}$ is a subset of $\Delta \mathcal{T}^{*}$.

Proof. The first statement follows immediately from Lemma 5.1. The second statement can be proved as follows: Let $d \in \Delta \mathcal{T}^{*}$. Then $d \mathcal{T}^{*}$ is a multiplicative coset of $\mathcal{T}^{*}$, and 
there exists $\beta \in \mathcal{T}^{*}$ such that $(1+p \beta) \mathcal{T}^{*}=d \mathcal{T}^{*}$. Since $d \in \Delta \mathcal{T}^{*}$, there are distinct elements $\alpha, \alpha^{\prime} \in \mathcal{T}^{*}$ such that $d=\alpha-\alpha^{\prime}$. Since for every $\gamma \in \mathcal{T}^{*}$ the elements $\alpha \gamma, \alpha^{\prime} \gamma$ are contained in $\mathcal{T}^{*}$, the set $d \mathcal{T}^{*}=\left\{d \gamma: \gamma \in \mathcal{T}^{*}\right\}$ is a subset of $\Delta \mathcal{T}^{*}$.

Let us now present the construction of $(v, k, k-1)$ disjoint difference families by Davis, Huczynska, and Mullen [8]. Since the authors of [8] proved the result in terms of external difference families, we will include a short proof that is analogous to the proof of Theorem 2.1. We remark that the theorem can also be proved using the results by Furino [9] or the Ferrero pairs by Buratti [6].

Theorem 5.3 ([8, Theorem 4.1]). Let $\mathcal{T}$ be the Teichmüller set of the Galois ring $G R\left(p^{2}, r\right)$, and let $\mathcal{T}^{*}=\mathcal{T} \backslash\{0\}$. The collection

$$
E=\left\{(1+p \alpha) \mathcal{T}^{*}: \alpha \in \mathcal{T}\right\} \cup p \mathcal{T}^{*}
$$

forms a near-complete $\left(p^{2 r}, p^{r}-1, p^{r}-2\right)$ disjoint difference family in the additive group of $G R\left(p^{2}, r\right)$.

Proof. We will first count the number of differences for the units of $\operatorname{GR}\left(p^{2}, r\right)$ and next for the non-invertible elements. Let $x, y \in \operatorname{GR}\left(p^{2}, r\right)^{*}$. Assume $x=u-u^{\prime}$, where $u, u^{\prime}$ are elements of the same coset $(1+p \alpha) \mathcal{T}^{*}$ of the Teichmüller group $\mathcal{T}^{*}$ for some fixed element $\alpha \in \mathcal{T}$. There exists $z \in \operatorname{GR}\left(p^{2}, r\right)$ so that $y=z x$. Hence, $y=z u-z u^{\prime}$ and $z u, z u^{\prime} \in\left(1+p \alpha^{\prime}\right) \mathcal{T}^{*}$ for some $\alpha^{\prime} \in \mathcal{T}$, and we have found a representation of $y$ as the difference of two distinct elements from the same set $\left(1+p \alpha^{\prime}\right) \mathcal{T}^{*}$. Since every difference for $y$ will also give us a difference for $x$, it follows that every unit will have the same number of differences. In each of the $p^{r}$ sets $(1+p \alpha) \mathcal{T}$ we have $\left(p^{r}-1\right)\left(p^{r}-2\right)$ differences, giving us a total of $p^{r}\left(p^{r}-1\right)\left(p^{r}-2\right)$ differences. From Lemma 5.2, we know that all these differences are units. Since there are $p^{r}\left(p^{r}-1\right)$ units in $\operatorname{GR}\left(p^{2}, r\right)$, each unit has

$$
\frac{p^{r}\left(p^{r}-1\right)\left(p^{r}-2\right)}{p^{r}\left(p^{r}-1\right)}=p^{r}-2
$$

representations as a difference from two distinct elements of the sets $(1+p \alpha) \mathcal{T}^{*}, \alpha \in \mathcal{T}$.

We now consider the non-invertible non-zero elements of $\operatorname{GR}\left(p^{2}, r\right)$, i. e. the elements of the set $p \mathcal{T}^{*}=\mathcal{I} \backslash\{0\}$. Since $\mathcal{I}$ is a group under addition, $\mathcal{I} \backslash\{0\}$ is a trivial $\left(p^{r}, p^{r}-1, p^{r}-2\right)$ difference set in $\mathcal{I}$. Hence, $\Delta p \mathcal{T}^{*}=\left(p^{r}-2\right)(\mathcal{I} \backslash\{0\})$. Combining these two results, we see that every non-zero element of $\operatorname{GR}\left(p^{2}, r\right)$ has $p^{r}-2$ differences in the sets of $E$.

Davis, Huczynska, and Mullen [8] remark that, since $p^{r}+1$ divides $p^{2 r}-1$, there is also a disjoint difference family with the same parameters in $\left(\mathbb{F}_{p^{2 r}},+\right)$ which can be constructed using Theorem 2.1. The authors ask whether the associated designs, i. e. the developments of these two disjoint difference families, are isomorphic. We will answer this question by showing that the designs are nonisomorphic in all but one case. This is our second main theorem.

Theorem 5.4. Let $C$ be $a\left(p^{2 r}, p^{r}-1, p^{r}-2\right)$ disjoint difference family in the additive group of the finite field $\left(\mathbb{F}_{p^{2 r}},+\right)$ constructed with Theorem 2.1, and let $E$ be a 
disjoint difference family with the same parameters in the additive group of the Galois ring $\left(G R\left(p^{2}, r\right),+\right)$ constructed with Theorem 5.3. The $2-\left(p^{2 r}, p^{r}-1, p^{r}-2\right)$ designs $\operatorname{dev}(C)$ and $\operatorname{dev}(E)$ are isomorphic if $p=3$ and $r=1$, and they are nonisomorphic in every other case.

To prove Theorem 5.4 we will consider four cases: First, we will examine the case $p=3$ and $r=1$, second, we will consider $p=2$ and $r=2$, third, we look at the case $p=2$ and $r \geq 3$, and last we will consider $p \geq 3$ and arbitrary $r$ (except $p=3$ and $r=1)$.

If $p=3$ and $r=1$, the 2-designs $\operatorname{dev}(C)$ and $\operatorname{dev}(E)$ are isomorphic. In this case $\operatorname{GR}(9,1) \cong \mathbb{Z}_{9}$ and $\left(\mathbb{F}_{9},+\right) \cong \mathbb{Z}_{3} \times \mathbb{Z}_{3}$. An isomorphism between $\operatorname{dev}(E)$ and $\operatorname{dev}(C)$ computed by Magma [5] is the map $f: \mathbb{Z}_{9} \rightarrow \mathbb{Z}_{3} \times \mathbb{Z}_{3}$ on the point set of $\operatorname{dev}(E)$ with

$$
\begin{aligned}
& 0 \mapsto(0,0), \quad 1 \mapsto(0,1), \quad 2 \mapsto(1,2), \quad 3 \mapsto(1,1), \quad 4 \mapsto(2,2) \\
& 5 \mapsto(2,0), \quad 6 \mapsto(1,0), \quad 7 \mapsto(2,1), \quad 8 \mapsto(0,2) .
\end{aligned}
$$

If $p=2$ and $r=2$, the designs $\operatorname{dev}(E)$ and $\operatorname{dev}(C)$ share the same block intersection numbers (together with their multiplicities), which we use in the proof of the remaining two cases. For both designs the block intersection numbers are 0 (1600 times), 1 (1440 times) and 2 (120 times). Hence, we solve this case by computing the automorphism groups of the designs with the help of Magma [5]: The automorphism group of $\operatorname{dev}(E)$ has order 384 while the automorphism group of $\operatorname{dev}(C)$ is of order 5760. If $\operatorname{dev}(E)$ and $\operatorname{dev}(C)$ were isomorphic, their automorphism groups would be the same. Hence, the two designs are nonisomorphic.

For the case $p=2$ and $r \geq 3$ and the case $p \geq 3$ (except $p=3$ and $r=1$ ) we will prove Theorem 5.4 similarly to Theorem 4.2 by showing that the block intersection numbers of $\operatorname{dev}(C)$ and $\operatorname{dev}(E)$ differ. We start by calculating the block intersection numbers of $\operatorname{dev}(C)$, that are the cyclotomic numbers of order $p^{r}+1$ in $\mathbb{F}_{p^{2 r}}$. As before, these cyclotomic numbers are uniform (see Proposition 4.3):

Corollary 5.5. Let $C$ be a $\left(p^{2 r}, p^{r}-1, p^{r}-2\right)$ disjoint difference family in the additive group of the finite field $\mathbb{F}_{p^{2 r}}$ constructed with Theorem 2.1. The 2- $\left(p^{2 r}, p^{r}-1, p^{r}-2\right)$ design dev $(C)$ has exactly three block intersection numbers, namely $p^{r}-2,0$ and 1.

Proof. Since $e=p^{r}+1$, we have $-1 \equiv p^{r}(\bmod e)$, and we are allowed to employ Proposition 4.3. From $s^{2}=p^{2 r}$ and $s \equiv 1(\bmod e)$ it follows that $s=-p^{r}$. Thus, $\eta=\left(-p^{r}-1\right) /\left(p^{r}+1\right)=-1$, and we get the cyclotomic numbers

$$
\begin{aligned}
(0,0)_{p^{r}+1} & =p^{r}-2, & & \\
(0, i)_{p^{r}+1}=(i, 0)_{p^{r}+1}=(i, i)_{p^{r}+1} & =0 & & \text { for } i \neq 0, \\
(i, j)_{p^{r}+1} & =1 & & \text { for } i \neq j \text { and } i, j \neq 0,
\end{aligned}
$$

that occur as the intersection numbers of $\operatorname{dev}(C)$.

Remark 3. Since $r$ divides $2 r$, the finite field $\mathbb{F}_{p^{2 r}}$ contains a unique subfield $\mathbb{F}_{p^{r}}$. Thus, the subgroup $C_{0}=\left\{\alpha^{i\left(p^{r}+1\right)}: i=0,1, \ldots, p^{r}-2\right\}$ of order $p^{r}-1$ of $\mathbb{F}_{p^{2 r}}^{*}$ that generates 
the disjoint difference family $C$ is the multiplicative group $\mathbb{F}_{p^{r}}^{*}$ of $\mathbb{F}_{p^{r}}$. Hence, it is clear that $\Delta C_{0}=\left(p^{r}-2\right) C_{0}$.

We now focus on the intersection numbers of $\operatorname{dev}(E)$ in the Galois ring $\operatorname{GR}\left(p^{2}, r\right)$. The following Lemma 5.6 in combination with Corollary 5.5 will finish the proof of Theorem 5.4.

Lemma 5.6. Let $E$ be a $\left(p^{2 r}, p^{r}-1, p^{r}-2\right)$ disjoint difference family in the additive group of the Galois ring $G R\left(p^{2}, r\right)$ constructed with Theorem 5.3. If $p=2$ and $r \geq 2$, the 2-( $\left(2^{2 r}, 2^{r}-1,2^{r}-2\right)$ design dev $(E)$ has a block intersection number 2 . If $p \geq 3$ (except the case $p=3$ and $r=1)$, the $2-\left(p^{2 r}, p^{r}-1, p^{r}-2\right)$ design dev $(E)$ has a block intersection number $N$ with $1<N<p^{r}-2$.

We prove Lemma 5.6 by a series of lemmas. We start by considering the Galois ring $\operatorname{GR}(4, r), r \geq 2$, i. e., we set $p=2$, and show that, in this case, 2 is a block intersection number of $\operatorname{dev}(E)$ if $r \geq 2$. Recall that in the case $p=2$, according to Lemma 4.6, -1 is a principal unit. Looking at the construction of our disjoint difference family $E$ we notice that for every block $(1+2 \alpha) \mathcal{T}^{*}, \alpha \in \mathcal{T}$, of $E$ its additive inverse $-(1+2 \alpha) \mathcal{T}^{*}, \alpha \in \mathcal{T}$, is also a block of $E$, and the two sets are disjoint. We also need the following result:

Lemma 5.7. In the Galois ring $G R(4, r), r \geq 2$, the Teichmüller set $\mathcal{T}$ is the set of all squares.

Proof. Let $x \in \operatorname{GR}(4, r)$. If $x \in \mathcal{I}$, say $x=p \alpha$ for some $\alpha \in \mathcal{T}$, then $x^{2}=p^{2} \alpha^{2}=0$. If $x$ is a unit, say $x=\alpha_{0}\left(1+p \alpha_{1}\right), \alpha_{0} \in \mathcal{T}^{*}, \alpha_{1} \in \mathcal{T}, \alpha_{0} \neq 0$, then $x^{2}=\alpha_{0}^{2}\left(1+p \alpha_{1}\right)^{2}=\alpha_{0}$ because each principal unit has order 2 .

To examine the block intersection numbers of $\operatorname{dev}(E)$ we use the well-known result that in a Galois ring of characteristic 4 the Teichmüller set is a relative difference set. We add a proof in Proposition 5.8. Let $G$ be a group of order $m n$ that contains a normal subgroup $N$ of order $n$. A $k$-subset $D$ of $G$ is called an $(m, n, k, \lambda)$-relative difference set in $G$ relative to $N$ if each element of $G \backslash N$ occurs exactly $\lambda$ times and the elements of $N$ do not occur in $\Delta D$.

Proposition 5.8. In $G R(4, r), r \geq 2$, the Teichmüller set $\mathcal{T}=\left\{0,1, \xi, \ldots, \xi^{2^{r}-2}\right\}$ is a $\left(2^{r}, 2^{r}, 2^{r}, 1\right)$-relative difference set in the additive group of $G R(4, r)$ relative to the maximal ideal $\mathcal{I}=2 G R(4, r)$.

Proof. This proof is similar to the one by Bonnecaze and Duursma [4, Lemmas 2 and 3]. From Lemma 5.1 we know that the difference $\beta-\beta^{\prime}$ of two Teichmüller elements $\beta, \beta^{\prime} \in \mathcal{T}$ is a unit if and only if $\beta \neq \beta^{\prime}$. Additionally, we know that each element $x$ of $\operatorname{GR}(4, r)$ has a unique 2-adic representation $x=\alpha_{0}+2 \alpha_{1}, \alpha_{0}, \alpha_{1} \in \mathcal{T}$. We consider the equation

$$
\alpha_{0}+2 \alpha_{1}=\beta-\beta^{\prime} .
$$


We choose $\beta$ and $\beta^{\prime}$ and fix thereby $\alpha_{0}$ and $\alpha_{1}$. Hence, (7) has $4^{r}$ solutions $\left(\alpha_{0}, \alpha_{1}, \beta, \beta^{\prime}\right)$. In the next step we consider the system of equations

$$
\begin{aligned}
\alpha_{0}^{2}+2 \alpha_{0} \alpha_{1}+\alpha_{1}^{2} & =\alpha_{0} \beta \\
\alpha_{1}^{2} & =\alpha_{0} \beta^{\prime} \\
\beta & =\beta^{\prime}, \quad \text { if } \alpha_{0}=0 .
\end{aligned}
$$

The system of equations has also $4^{r}$ solutions $\left(\alpha_{0}, \alpha_{1}, \beta, \beta^{\prime}\right)$ : It has $2^{r}$ solutions if $\alpha_{0}=0$, namely $(0,0, \beta, \beta)$ for arbitrary $\beta$, and it has $2^{r}\left(2^{r}-1\right)$ solutions if $\alpha_{0} \neq 0$ : In this case, we can chose $\alpha_{0} \in \mathcal{T}^{*}, \alpha \in \mathcal{T}$ arbitrarily and obtain unique solutions for $\beta$, $\beta^{\prime}$, namely

$$
\begin{aligned}
\beta & =\alpha_{0}^{-1}\left(\alpha_{0}+\alpha_{1}\right)^{2}, \\
\beta^{\prime} & =\alpha_{0}^{-1} \alpha_{1}^{2} .
\end{aligned}
$$

It is easy to check that the solutions to the system of equations also solve (7). Hence, each unit $\alpha_{0}+2 \alpha_{1}, \alpha_{0} \neq 0$, can be uniquely represented as the difference of two Teichmüller elements $\beta, \beta^{\prime}$ as described in (8) and (9).

Proposition 5.8 implies

Corollary 5.9. In $G R(4, r), r \geq 2$, we have for each $\alpha \in \mathcal{T}$ that

$$
\Delta(1+2 \alpha) \mathcal{T}^{*}=G R(4, r) \backslash\left(\mathcal{I} \cup(1+2 \alpha) \mathcal{T}^{*} \cup-(1+2 \alpha) \mathcal{T}^{*}\right),
$$

and each element of $G R(4, r) \backslash\left(\mathcal{I} \cup(1+2 \alpha) \mathcal{T}^{*} \cup-\left(1+2 \alpha \mathcal{T}^{*}\right)\right.$ has multiplicity 1 in this multiset.

Proof. According to Proposition 5.8 the Teichmüller set $\mathcal{T}$ is a $\left(2^{r}, 2^{r}, 2^{r}, 1\right)$ relative difference set in the additive group of $\operatorname{GR}(4, r)$ relative to $\mathcal{I}$. Hence, by removing 0 from $\mathcal{T}$ to obtain $\mathcal{T}^{*}$ we remove differences of the type $\mathcal{T}^{*}-0=\mathcal{T}^{*}$ and $0-\mathcal{T}^{*}=-\mathcal{T}^{*}$ from our set of differences.

These results enable us to determine an intersection number of $\operatorname{dev}(E)$. For our purpose the following Lemma 5.10 suffices. A more detailed analysis of the differences and sums in the Teichmüller set in GR $(4, r)$, that includes the following result in a slightly different way, is given by [11, Section III.-C.] and Bonnecaze and Duursma [4, Theorem 1]. Arguments of this type have also been used by Ghinelli and Jungnickel [10] and Resmini, Ghinelli, and Jungnickel [15] in the theory of difference sets to obtain ovals in the development of a difference set and by Pott and Zhou [14] to construct Cayley graphs .

Lemma 5.10. In $G R(4, r), r \geq 2$, an element $s$ of the multiset $\Delta_{+}(1+2 \alpha) \mathcal{T}^{*}$ has multiplicity 2 if $s$ is a unit and multiplicity 1 if $s \in \mathcal{I} \backslash\{0\}$.

Proof. Let $\beta, \gamma$ be two distinct elements of the Teichmüller group $\mathcal{T}^{*}$. Since $2 \mathcal{T}=\mathcal{I}$, it follows that $\beta+\beta=2 \beta \neq 2 \gamma=\gamma+\gamma$. Hence, the elements of $\mathcal{I} \backslash\{0\}$ are represented 
once as the sum of two elements of $\mathcal{T}^{*}$. We now consider sums of the type $\beta+\gamma, \beta \neq \gamma$. It is clear that each sum $s=\beta+\gamma$ has at least two representations: $\beta+\gamma$ and $\gamma+\beta$. To prove that there are no more than those two representations we assume that there exist elements $\beta^{\prime}, \gamma^{\prime} \in \mathcal{T}^{*}, \beta^{\prime}, \gamma^{\prime} \notin\{\beta, \gamma\}$ such that $\beta+\gamma=\beta^{\prime}+\gamma^{\prime}$. This is equivalent to $\beta-\beta^{\prime}=\gamma^{\prime}-\gamma$. However, according to Corollary 5.9 all the differences of two distinct elements of $\mathcal{T}^{*}$ are distinct. Consequently, $\beta+\gamma \neq \beta^{\prime}+\gamma^{\prime}$.

Corollary 5.9 and Lemma 5.10 lead us to the following statement about the block intersection numbers in $\operatorname{dev}(E)$ :

Lemma 5.11. In $G R(4, r), r \geq 2$, let $E_{\alpha}=(1+2 \alpha) \mathcal{T}^{*}, \alpha \in \mathcal{T}$, and let $d \in G R(4, r)$. Then

$$
\begin{aligned}
\left|\left(E_{\alpha}+d\right) \cap E_{\alpha}\right| & = \begin{cases}1, & \text { if } d \in \Delta E_{\alpha}, \\
0, & \text { in any other case, }\end{cases} \\
\left|\left(E_{\alpha}+d\right) \cap-E_{\alpha}\right| & = \begin{cases}2, & \text { if } d \in\left(\Delta_{+}-E_{\alpha}\right) \backslash \mathcal{I}, \\
1, & \text { if } d \in \mathcal{I} \backslash\{0\}, \\
0, & \text { in any other case. }\end{cases}
\end{aligned}
$$

Since $(1+2 \alpha) \mathcal{T}^{*}+d, \alpha \in \mathcal{T}, d \in \operatorname{GR}(4, r)$, and $-(1+2 \alpha) \mathcal{T}^{*}$ are blocks of the design $\operatorname{dev}(E)$, it follows that $\operatorname{dev}(E)$ contains blocks that intersect in two elements. According to Corollary 5.5 however, the design $\operatorname{dev}(C)$ has block intersection numbers $2^{r}-2,0$ and 1 , and $2^{r}-2>2$ if $r \geq 3$. We conclude that the combinatorial designs $\operatorname{dev}(C)$ and $\operatorname{dev}(E)$ are nonisomorphic if $p=2$ and $r \geq 3$.

Now, let $p$ be an odd prime.

Lemma 5.12. In $G R\left(p^{2}, r\right)$, $p$ odd, we have $\mathcal{T}^{*}=-\mathcal{T}^{*}$.

Proof. According to Lemma $4.6,-1 \in \mathcal{T}^{*}$ if $p$ is odd.

Hence, the Teichmüller group consists of pairs of an element and its additive inverse and we can write

$$
\mathcal{T}^{*}=\left\{1, \xi, \xi^{2}, \ldots, \xi^{\left(p^{r}-3\right) / 2},-1,-\xi,-\xi^{2}, \ldots,-\xi^{\left(p^{r}-3\right) / 2}\right\} .
$$

From this notation we can deduce

Lemma 5.13. In $G R\left(p^{2}, r\right), p$ odd, a difference d of two distinct elements of $\mathcal{T}^{*}$ occurs at least twice in $\Delta \mathcal{T}^{*}$, except if $d \in 2 \mathcal{T}^{*}$, then $d$ occurs at least once in $\Delta \mathcal{T}^{*}$. Consequently, a difference $d$ has odd multiplicity in $\Delta \mathcal{T}^{*}$ if and only if $d \in 2 \mathcal{T}^{*}$.

Proof. Let $d=\alpha-\alpha^{\prime} \in \Delta \mathcal{T}^{*}$ be the difference of two arbitrary elements of the Teichmüller group $\mathcal{T}^{*}$. According to Lemma 5.12, the elements $-\alpha,-\alpha^{\prime}$ are also in $\mathcal{T}^{*}$. Hence, $-\alpha^{\prime}-(-\alpha)=d$ is another representation of $d$ as the difference of two elements from $\mathcal{T}^{*}$. However, those two representations are the same, if $\alpha^{\prime}=-\alpha$. Then $d=2 \alpha$, 
and it is not guaranteed that $d$ has more than this single representation. It could, however, happen, that there exist more distinct pairs $\left(\beta_{1}, \beta_{1}^{\prime}\right), \ldots,\left(\beta_{\ell}, \beta_{\ell}^{\prime}\right), \beta_{1}, \beta_{1}^{\prime}, \ldots, \beta_{\ell}, \beta_{\ell}^{\prime} \in$ $\mathcal{T}^{*} \backslash\left\{\alpha, \alpha^{\prime}\right\}$ with $\beta_{i}-\beta_{i}^{\prime}=-\beta_{i}^{\prime}-\left(-\beta_{i}\right)=d$. Then, $d$ has $2 \ell+2$ representations as a difference if $d \notin 2 \mathcal{T}^{*}$ and $2 \ell+1$ representations if $d \in \mathcal{T}^{*}$.

Corollary 5.14. In $G R\left(p^{2}, r\right), p$ odd (except $p=3$ and $\left.r=1\right)$, let $d \in\left(\Delta \mathcal{T}^{*} \backslash 2 \mathcal{T}^{*}\right)$. Then, the block intersection number $\left|\mathcal{T}^{*} \cap\left(\mathcal{T}^{*}+d\right)\right| \geq 2$.

Proof. The statement follows from Lemma 5.13: Let $d=\alpha-\alpha^{\prime}$ for some distinct $\alpha, \alpha^{\prime} \in$ $\mathcal{T}^{*}, \alpha^{\prime} \neq-\alpha$. Then $d$ occurs at least twice in $\Delta \mathcal{T}^{*}$. In the case $p=3$ and $r=1$, the Teichmüller group contains only two elements, namely 1 and -1 . Hence, there are no $\alpha, \alpha^{\prime} \in \mathcal{T}^{*}$ with $\alpha \neq-\alpha^{\prime}$. So, we need to exclude this case.

To finish our proof of Lemma 5.6 we need to show that the block intersection numbers greater than 1 from Corollary 5.14 are less than $p^{r}-2$.

Lemma 5.15. In $G R\left(p^{2}, r\right), p$ odd (except $p=3$ and $r=1$ ), there is no difference $d \in \Delta \mathcal{T}^{*}$ with multiplicity $p^{r}-2$ in $\Delta \mathcal{T}^{*}$.

Proof. Assume there is an element $d \in \Delta \mathcal{T}^{*}$ with multiplicity $p^{r}-2$. We know from Lemma 5.2 that $\Delta \mathcal{T}^{*}$ is the union of whole cosets of $\mathcal{T}^{*}$ and that the elements of the same coset have the same multiplicity. Counting multiplicities, $\Delta \mathcal{T}^{*}$ contains $\left(p^{r}-1\right)\left(p^{r}-2\right)$ elements. Hence, if $d$ has multiplicity $p^{r}-2$, every $d^{\prime}$ from the same coset as $d$ will also have multiplicity $p^{r}-2$. Since $\mathcal{T}^{*}$ and its cosets contain $p^{r}-1$ elements, this means that $\Delta \mathcal{T}^{*}$ consists of $p^{r}-2$ times the same coset. Obviously, $p^{r}-2$ is odd. From Lemma 5.13 we know, that an element $d \in \mathcal{T}^{*}$ only has odd multiplicity if $d \in 2 \mathcal{T}^{*}$. Thus, we have $\Delta \mathcal{T}^{*}=\left(p^{r}-2\right)\left(2 \mathcal{T}^{*}\right)$. Now, let $\alpha$ be an arbitrary element of $\mathcal{T}^{*}$. Then $\alpha-1 \in \Delta \mathcal{T}^{*}$, and there is an element $\beta \in \mathcal{T}^{*}$ such that $\alpha-1=2 \beta$. However, since $-1 \in \mathcal{T}^{*}$, the element $\alpha+1$ is also in $\Delta \mathcal{T}^{*}$, and we have $\alpha+1=2 \beta+2=2(\beta+1)$. Since $p \geq 3$, the element 2 is a unit, and thus $\beta+1 \in \mathcal{T}^{*}$. Consequently, we have $\Delta \mathcal{T}^{*}=\left(p^{r}-2\right) \mathcal{T}^{*}$.

In other words, $\mathcal{T}^{*}$ needs to be an (additive) $\left(p^{r-1}, p^{r-2}, p^{r-2}\right)$ difference set in $\mathcal{T}=$ $\mathcal{T}^{*} \cup\{0\}$. This is only the case if $\mathcal{T}$ forms an additive group. It is clear, however, that this is not the case: Since $1 \in \mathcal{T}$, this would mean that $p \in \mathcal{T}$, but $p$ is not a unit. Hence, there is no $d \in \mathcal{T}^{*}$ with multiplicity $p^{r}-2$.

Lemma 5.15 immediately gives us the following result.

Corollary 5.16. In $G R\left(p^{2}, r\right), p$ odd (except $p=3$ and $r=1$ ), the block intersection number $\left|\mathcal{T}^{*} \cap\left(\mathcal{T}^{*}+d\right)\right|<p^{r}-2$ for all $d \in \Delta \mathcal{T}^{*}$.

By combining Corollary 5.14 and Corollary 5.16 we see that for odd $p$ (except $p=3$ and $r=1)$ there exists an element $d \in \operatorname{GR}\left(p^{2}, r\right)$ such that the intersection number of the blocks $\mathcal{T}^{*}$ and $\mathcal{T}^{*}+d$ of $\operatorname{dev}(E)$ is greater than 1 and less than $p^{r}-2$. This concludes the proof of Lemma 5.6. 


\section{References}

\section{Conclusion}

In this paper, we solve the isomorphism problem for two pairs of near-complete $(v, k, k-1)$ disjoint difference families in Galois rings and finite fields. However, there exist many more constructions of difference families, and it is a natural question to ask whether their associated designs are nonisomorphic. Hence, we leave to future work the task to solve the isomorphism problem for more disjoint difference families.

Moreover, it would be nice to have a general powerful construction of difference families for which one can show that (almost) all their candidates are nonisomorphic. For parameters $(v, k, k-1)$, the construction presented by Buratti [6] seems to be powerful, and it will be interesting to check if this construction leads to nonisomorphic designs.

\section{References}

[1] R. J. R. Abel and M. Buratti. Difference Families. In: Handbook of Combinatorial Designs. Ed. by C. J. Colbourn and J.H. Dinitz. 2nd ed. Boca Raton: Chapman \& Hall/CRC Press, 2006, pp. 392-410.

[2] L. D. Baumert, W. H. Mills, and R. L. Ward. Uniform Cyclotomy. In: J. Number Theory 14.1 (1982), pp. 67-82.

[3] T. Beth, D. Jungnickel, and H. Lenz. Design Theory. 2nd ed. Cambridge: Cambridge University Press, 1999.

[4] A. Bonnecaze and I. M. Duursma. Translates of Linear Codes over $\mathbb{Z}_{4}$. In: IEEE Trans. Inform. Theory 43.4 (1997), pp. 1218-1230.

[5] W. Bosma, J. Cannon, and C. Playoust. The Magma algebra system. I. The user language. In: J. Symbolic Comput. 24.3-4 (1997), pp. 235-265.

[6] M. Buratti. On disjoint $(v, k, k-1)$ difference families. In: ArXiv e-prints (May 2017). arXiv: 1705.04844 [math.CO].

[7] Y. Chang and C. Ding. Constructions of external difference families and disjoint difference families. In: Des. Codes Cryptogr. 40.2 (2006), pp. 167-185.

[8] J. A. Davis, S. Huczynska, and G. L. Mullen. Near-complete external difference families. In: Des. Codes Cryptogr. 84 (2017), pp. 415-424.

[9] S. Furino. Difference families from rings. In: Discrete Math. 97.1-3 (1991), pp. 177190.

[10] D. Ghinelli and D. Jungnickel. Finite projective planes with a large abelian group. In: Surveys in combinatorics, 2003 (Bangor). Vol. 307. London Math. Soc. Lecture Note Ser. Cambridge Univ. Press, Cambridge, 2003, pp. 175-237. 


\section{References}

[11] A. R. Hammons Jr., P. V. Kumar, A. R. Calderbank, N. J. A. Sloane, and P. Solé. The $\mathbb{Z}_{4}$-Linearity of Kerdock, Preparata, Goethals, and Related Codes. In: IEEE Trans. Inform. Theory 40.2 (1994), pp. 301-319.

[12] K. Momihara. Disjoint difference families from Galois rings. In: Electron. J. Combin. 24.3 (2017), P3.23.

[13] S.-L. Ng and M. B. Paterson. Disjoint difference families and their applications. In: Des. Codes Cryptogr. 78.1 (2016), pp. 103-127.

[14] A. Pott and Y. Zhou. Cayley Graphs of Diameter Two from Difference Sets. In: J. Graph Theory 85.2 (2017), pp. 533-544.

[15] M. J. de Resmini, D. Ghinelli, and D. Jungnickel. Arcs and Ovals from abelian Groups. In: Des. Codes Cryptogr. 26.1-3 (2002), pp. 213-228.

[16] Z.-X. Wan. Lectures on Finite Fields and Galois Rings. Singapore: World Scientific, 2003.

[17] R. M. Wilson. Cyclotomy and Difference Families in Elementary Abelian Groups. In: J. Number Theory 4.1 (1972), pp. 17-47.

[18] J. Zwanzger. Computergestützte Suche nach optimalen linearen Codes über endlichen Kettenringen unter Verwendung heuristischer Methoden. PhD thesis. Bayreuth, 2011. URL: https://epub.uni-bayreuth.de/331/2/dissertation.pdf. 